\title{
Association Between Using Multimedia and Overweight Obesity Among the Preparatory School Students
}

\author{
Abeer Abd El-Aziz Madian \& Marwa Mohamed Ahmed. \\ Assistant Prof. of Community Health Nursing Dept, Faculty of Nursing, Damanhour University, Egypt. \\ Lecturer of Pediatric Nursing Department, Faculty of Nursing, Damanhour University, Egypt.
}

\begin{abstract}
The Aim: is to assess association between using multimedia and overweight/obesity among the preparatory school students. Research design: A cross sectional design was used. Sample: using stratified multi-stage sampling technique, a total number of 900 students constituted the subjects of the study. Setting: the study was conducted at the governmental preparatory schools in Damanhour city Tool: Structured questionnaire was developed by the researcher after reviewing literature and used to collect data. Results: the results revealed that half of students were overweight and $16 \%$ of them were obese. The majority of overweight and obese students had personal computers and closely two thirds of them had home internet. Also, it was clear that, approximately half of all overweight/obese students and the majority of them were using computer and internet daily in school days and summer holiday respectively. However, less than two thirds of the studied students who were overweight and obese had health problems due to using computer and watching TV, mainly vision problems. The present study Concluded that, there was an association between using multimedia and overweight / obesity among the preparatory school students So, it is Recommended to develop and implement a school-based programs as these programs effectively diminish the consumed time for watching TV and PCs.
\end{abstract}

\section{Keywords: Multimedia, School Students, Overweight, Obesity, Computer Using \& TV Viewing.}

\section{Introduction}

Adolescent population and their health are a very special issue and focus of attention globally for various reasons, as it is a period of rapid physical and psychological growth. Also, it provokes changes in social functioning and increasing demands from and influence of peers, school and wider society. This developmental period allows for the testing of new behaviors and roles (WHO, 2009).

In latest years, automated games, home computers, and the Internet have implicit vital abode in our lives (Barton, 2010 \& Bener et al., 2010).With the amplified role of home computers in offspring's survives, it increased apprehension about how children may be pretentious. Therefore, time spent on home computers may displace other activities that have more developmental value (WHO, 2011 \& Straker et al., 2009).

Obesity is caused by an imbalance between energy consumed and energy expended, with excess energy stored as fat. Childhood obesity is a noteworthy community health tricky upsetting nearly $17 \%$ of American youth. Over weight / Obese children are more probable to have physical, emotional and psychological health glitches, such as type 2 diabetes, cardiovascular disease, and depression. The health risks associated with childhood over weight and obesity persist into adulthood and include arthritis, stroke, liver disease, cancer and lower life expectancy (Tewfik, 2011).

The cause of adolescents' over weight / obesity is certainly debated. Some researchers have pointed to socioeconomic factors, poor eating habits, soft drink consumption and lack of physical exercises, while advocacy groups have accused mass media as the offender for advertising junk food to offspring. Children spend regular of 5.5 hours per day using numerous media and are bare to an average of one food profitable every 5 minutes, 40,000 television advertisements annually. Most of those advertisements are for sweetie, high sugar cereals, and fast food (Anuradha et al., 2015\& Wieting, 2008)

Progress in computer technology designate that this danger factor is going to increase additional for future generations (The Royal Australasian College of Physicians, 2004). Plentiful studies have recognized the influence of the content of TV and the Internet on adolescents' lifestyle choices; these range from the consequence of popular TV characters' poor eating habits to high calorie food marketing directed at children on both the TV and Internet. A large body of literature has documented the effect of the time spent watching TV or using the internet on children, in terms of, assuming sedentary lifestyle behaviors associated with 
obesity (Singh et al., 2010 \& Manyanga et al., 2014).

Additional research has shown that: "Plummeting television viewing and computer use may have an important role in avoiding obesity and in lowering BMI in young children and adolescents" (Rosen et al., 2014\& Utter et al., 2006). Moreover, several researchers decided that $\mathrm{TV}$ viewing time may be accompanying with a loss of life that is analogous to other major chronic disease risk aspects such as physical inactivity and obesity (Blackwell, 2015).

\section{Significant of study}

Overweight / obesity have become important public health problems in children and adolescent because they are associated with hypertension, dyslipidemia, high cholesterol and impaired glucose metabolism that have an impact on the physical health and can lead to an increase in the risk of early illness and death in later life. The prospective sponsors to the problem of adolescent's obesity are manifold and complex, increasing television viewing and computer used in offspring has been implicated (Talat et al., 2016 \&Mota et al., 2006). Obesity during childhood and adolescence is significant element of whether an individual will become obese as an adult. It has been found that $30 \%$ of girls and $10 \%$ of boys who were obese as children were also obese adults the risk for perseverance is greater in adolescence than in childhood. In Egypt (2014) the occurrence of overweight among boys and girls was $24.5 \%$ and $26.9 \%$ correspondingly, while the prevalence of obesity among boys and girls was $10.5 \%$ and $9.5 \%$ correspondingly among age group 5-19 years (UNICEF, 2015).

\section{Aim of study}

to determine association between using multimedia and overweight / obesity among the preparatory school students.

Research question: The research question for this study was:

1- What is the association between using multimedia and overweight/ obesity among the preparatory school students?

\section{Material \& Methods Design}

A cross sectional descriptive design was approved to conducted this study.

Setting

The study was implemented at the governmental preparatory schools in Damanhour city, namely Taha Hussein and El-Gomohria boys' preparatory schools. Besides, Shobra and El- Taaoan girls' preparatory schools.

\section{Subjects}

The study included 900 preparatory school students both boys and girls .By using equal allocation method, 450 boys were selected from the previous mentioned schools, and the same number for girls, from a list of classes by grades. The students were included from the first, second and third grades which were randomly selected and who accepted to share in the present study and were compatible with the measures for selection.

\section{Sampling technique}

By using stratified multi-stage sampling technique; El-Beheira Governorate is composed of 18 educational administrative departments. First stage, one educational administrative department (Damanhour) was randomly selected using lottery to be the place of study. Secondly, by equal allocation for gender, four governmental preparatory schools were randomly selected; two for boys and two for girls to represent the setting of study.

\section{Sample size}

According to central agency of public mobilization and statistics of El-Beheira governorate, books of census and statistics, Detailed Results 2014, the total number of preparatory students enrolled in Damanhour educational administrative department was (16.504 for girls and 15.117 for boys). (Central agency of public mobilization \& statistics of El-Beheira governorate,2014) A sample size of 900 was found required to detect prevalence of overweight and obesity among adolescents using computer and media $=37.8 \%$, using alpha error $=5 \%$, Precision $=7 \%$ and design effect $=2$. Inclusion criteria: The study sample was selected according to the following criteria:

- Students free from chronic diseases

- Not receiving for long acting medical treatment Tool for data collection

Structured questionnaire was developed after reviewing literature and used by the researchers in order to collect the necessary data from students at the selected schools. The questionnaire consists of five parts

Part I: This section consists of sociodemographic data includes age, sex, mothers' and fathers' educational level and occupation , income, and crowding index.

\section{Part II: Anthropometric valuation}

Weight in kilograms and height in centimeters were measured for all students included in the study. Weight was noted to the nearest $0.5 \mathrm{~kg}$, height was noted to the nearest $0.1 \mathrm{~cm}$ and body mass index (BMI) was intended. Then, likened 
with the reference value to recognize overweight or obesity among students as following according to Centres for disease control $\&$ prevention, 2015)

- Less than 5th percentile (underweight)

- 5th percentile to less than 85th percentile (normal weight)

- 85th to less than 95th percentile (overweight)

- Equal to or greater than 95th percentile (obesity)

Part III: It consists of items to assess students' resorted resated practices regarding using multimedia as:

- presence of computer and internet at home , frequency of using computer and internet in school days and in summer holiday, frequency of watching television.

Part IV: This section consists of students' reported practice regarding the physical exercises as: frequency of hours, practicing physical exercise per week and types of exercises.

Part V: This section consists of questions regarding eating habits as: number of meals/day, taking breakfast, frequency of taking foods or beverages during playing computer or watching television, frequency of eating the basic/daily meals in front of the television or computer and frequency of eating junk food.

- Also, this part included data related to the studied students about health problems due to playing computer and watching television.

- Hours spent sleeping per/day.

Methods

- Permission to conduct the study was obtained from director of the educational administrative department of Damanhour City.

- Selection of schools represents setting of study for both boys and girls. Then, permission from directors of the selected schools was obtained.

- All students enrolled at the first, second and third grades were included in the study after explaining the purpose of study.

Data collection

- Tool of data collection was designed based on recent relevant literature and was tested for its content validity by a jury of 3 experts at the same field.

- Tool reliability was tested by Cronbach alpha test, its results was 0.756 which indicates an accepted reliability of the tool.

- The pilot study was conducted on 90 students (10\% from total sample) from a preparatory school other than those included in the sample in order to ascertain its clarity and feasibility (New
Taawon girls school and King Faisal boys school).

- The purpose of pilot the study was clarified to each respondent and a questionnaire was given to the particular respondents. They were also encouraged to complete the questionnaire in the presence of the researchers.

- Each sheet took 25-30 minutes to be answered. Data was collected throughout the academic year 2015- 2016. This started at September/2015 till May 2016.

- (7-10sheets /day\&2day/week)

Ethical considerations

The questionnaire sheet was explained to the participants, oral consent was obtained from each student. Privacy was maintained during the process of data collecting. Confidentiality of students' response was guaranteed during the study.

Statistical analysis

After assortment of data, they were oblique and shifted into especially designed formats to be appropriate for computer feeding. Following data entry, scrutiny and verifying processes were conceded to evade any faults during data entry.

- Data was investigated using PC with Statistical Package for Social Sciences (SPSS) version 16.0.

- The level of significance designated for this study was $\infty=0.05$.

- The subsequent statistical measures were used

A- Descriptive statistics

Count and percentage: Used for recitation and succinct categorical variables, arithmetic means, Standard deviation (SD) and range used as measures of central tendency and dispersion correspondingly to recapitulate quantitative data.

\section{B-Analytical statistics:}

- Stepwise Logistic Regression using Forward Conditional Method was applied for identifying the possible risk factors for obesity (obese \&overweight) and their virtual belongings. 


\section{Results}

Table (1): Distribution of the studied students according to their personal characteristics. $(\mathrm{N}=900)$.

\begin{tabular}{|l|c|c|}
\hline \multirow{2}{*}{ Students' characteristics } & \multicolumn{2}{|c|}{ Studied sample (n=900) } \\
\cline { 2 - 3 } & \multicolumn{2}{|c|}{ No } \\
\hline Age (years) & \multicolumn{2}{|c|}{ \% } \\
\hline $11-$ & 174 & 19.3 \\
\hline $13-$ & 555 & 1.7 \\
\hline $15-16$ & 171 & 19.0 \\
\hline Min. - Max. & \multicolumn{2}{|c|}{$13.36 \pm 1.06$} \\
\hline Mean \pm SD. & \multicolumn{2}{|c|}{} \\
\hline Sex & 450 & 50.0 \\
\hline Male & 450 & 50.0 \\
\hline Female & \multicolumn{2}{|c|}{} \\
\hline Academic year & 564 & 62.7 \\
\hline Grade One & 186 & 20.7 \\
\hline Grade two & 150 & 16.6 \\
\hline Grade three & \multicolumn{2}{|c|}{} \\
\hline
\end{tabular}

Table (2): Distribution of the studied students according to their parents' socio-demographic data $\quad(\mathrm{N}=900)$.

\begin{tabular}{|c|c|c|}
\hline \multirow{2}{*}{ Parents' socio-demographic data } & \multicolumn{2}{|c|}{ Studied sample $(\mathrm{n}=900)$} \\
\hline & No & $\%$ \\
\hline \multicolumn{3}{|l|}{ Father`s Education } \\
\hline Illiterate & 41 & 4.6 \\
\hline Primary & 13 & 1.4 \\
\hline Preparatory & 117 & 13.0 \\
\hline Secondary & 178 & 19.8 \\
\hline University & 551 & 61.2 \\
\hline \multicolumn{3}{|l|}{ Father's occupation } \\
\hline Not working & 23 & 2.5 \\
\hline Handicraft & 89 & 9.9 \\
\hline Employee & 376 & 41.8 \\
\hline Free business & 277 & 30.8 \\
\hline Professional & 135 & 15.0 \\
\hline \multicolumn{3}{|l|}{ Mother`s Education } \\
\hline Illiterate & 75 & 8.3 \\
\hline Primary & 27 & 3.0 \\
\hline Preparatory & 97 & 10.8 \\
\hline Secondary & 149 & 16.6 \\
\hline University & 552 & 61.3 \\
\hline \multicolumn{3}{|l|}{ Mother`s occupation } \\
\hline House Wife & 516 & 57.3 \\
\hline Employee & 319 & 35.4 \\
\hline Free business & 15 & 1.7 \\
\hline Professional & 50 & 5.6 \\
\hline \multicolumn{3}{|l|}{ Family Income } \\
\hline less than $250 \mathrm{LE}$ & 27 & 3.0 \\
\hline $250-$ & 41 & 4.6 \\
\hline $500-$ & 119 & 13.2 \\
\hline More than $1000 \mathrm{LE}$ & 713 & 79.2 \\
\hline Crowding index & \multicolumn{2}{|c|}{$\frac{1}{1.63 \pm 0.85}$} \\
\hline
\end{tabular}




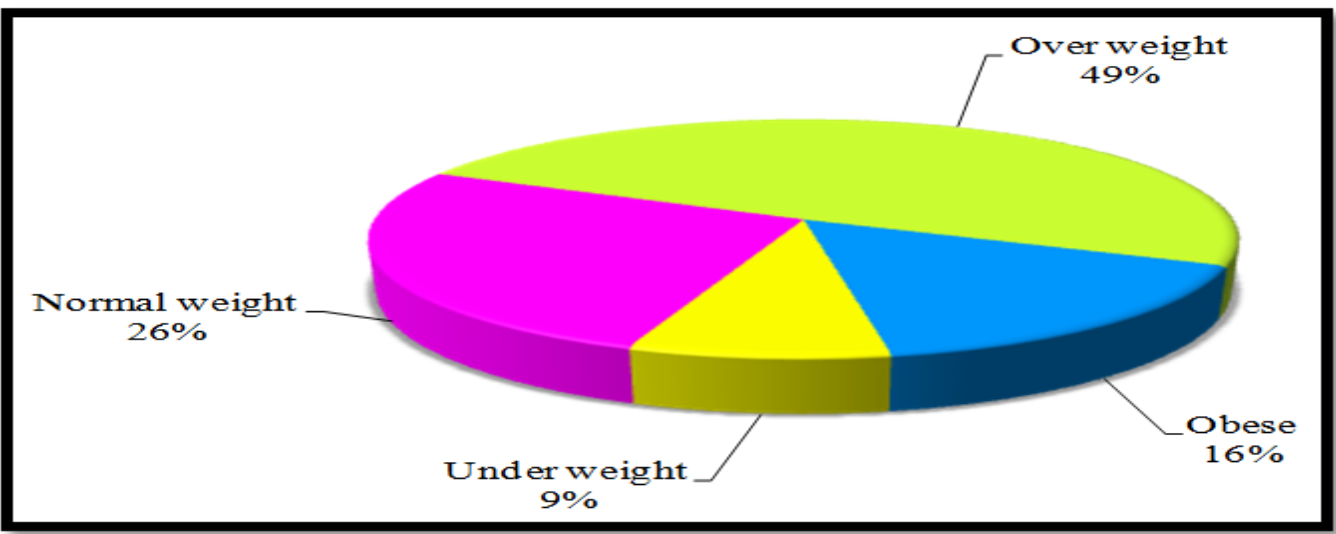

Figure (1): Total students' body mass index (BMI) (No=900).

Table (3): Distribution of overweight and obese students according to their presence of computer and internet at home. $($ No $=\mathbf{5 8 5})$.

\begin{tabular}{|c|c|c|c|c|c|c|}
\hline \multirow{3}{*}{ Items } & \multicolumn{4}{|c|}{ BMI } & \multirow{2}{*}{\multicolumn{2}{|c|}{$\begin{array}{c}\text { Total } \\
(\mathbf{N}=585)\end{array}$}} \\
\hline & \multicolumn{2}{|c|}{$\begin{array}{c}\text { Over weight } \\
(N=441)\end{array}$} & \multicolumn{2}{|c|}{$\begin{array}{c}\text { Obese } \\
(\mathrm{N}=144)\end{array}$} & & \\
\hline & No. & $\%$ & No. & $\%$ & No. & $\%$ \\
\hline \multicolumn{7}{|l|}{ Presence of home computer } \\
\hline Yes & 432 & 73.9 & 144 & 24.6 & 576 & 98.5 \\
\hline No & 9 & 1.5 & 0 & 0.0 & 9 & 1.5 \\
\hline If no, place of using computer & $\mathrm{n}=9$ & & $\mathbf{n}=\mathbf{0}$ & & $(\mathbf{n}=9)$ & \\
\hline School & 1 & 11 & 0 & 0.0 & 1 & 11 \\
\hline Relatives and neighbors & 4 & 44.5 & 0 & 0.0 & 4 & 44.5 \\
\hline Net coffee (Cyber) & 4 & 44.5 & 0 & 0.0 & 4 & 44.5 \\
\hline \multicolumn{7}{|l|}{ Presence of home internet } \\
\hline Yes & 251 & 42.9 & 113 & 19.3 & 364 & 62.2 \\
\hline No & 190 & 32.5 & 31 & 5.3 & 221 & 37.8 \\
\hline
\end{tabular}

Table (4): Distribution of overweight and obese students according to their using of computer and internet. $($ No $=\mathbf{5 8 5})$.

\begin{tabular}{|c|c|c|c|c|c|c|}
\hline \multirow{3}{*}{ Items } & \multicolumn{4}{|c|}{ BMI } & \multirow{2}{*}{\multicolumn{2}{|c|}{$\begin{array}{c}\text { Total } \\
(\mathbf{N}=\mathbf{5 8 5})\end{array}$}} \\
\hline & \multicolumn{2}{|c|}{$\begin{array}{l}\text { Over weight } \\
(\mathrm{N}=441)\end{array}$} & \multicolumn{2}{|c|}{$\begin{array}{c}\text { Obese } \\
(N=144)\end{array}$} & & \\
\hline & No. & $\%$ & No. & $\%$ & No. & $\%$ \\
\hline \multicolumn{7}{|l|}{ Using computer and the internet in school days } \\
\hline Daily & 175 & 29.9 & 84 & 14.4 & 259 & 44.3 \\
\hline Weekend(2days / week) & 81 & 13.8 & $\overline{0}$ & 0.0 & 81 & 13.8 \\
\hline 5 days/ week (week days) & 185 & 31.6 & 60 & 10.3 & 245 & 41.9 \\
\hline $\begin{array}{l}\text { Daily number of hours of using computer } \\
\text { and internet in school day }\end{array}$ & $\mathbf{n}=\mathbf{1 7 5}$ & & $n=84$ & & $(n=259)$ & \\
\hline$<4$ hours & 23 & 13.1 & 12 & 14.3 & 35 & 13.5 \\
\hline$\geq 4$ hours & 152 & 86.9 & 72 & 85.7 & 224 & 86.5 \\
\hline $\begin{array}{l}\text { Number of hours of using computer and } \\
\text { internet in school day during 5days/week }\end{array}$ & $n=185$ & & $n=60$ & & $(n=245)$ & \\
\hline$<4$ hours & 35 & 18.9 & 5 & 8.3 & 40 & 16.3 \\
\hline$\geq 4$ hours & 150 & 81.1 & 55 & 91.7 & 205 & 83.7 \\
\hline
\end{tabular}




\begin{tabular}{|c|c|c|c|c|c|c|}
\hline \multirow{3}{*}{ Items } & \multicolumn{4}{|c|}{ BMI } & \multirow{2}{*}{\multicolumn{2}{|c|}{$\begin{array}{c}\text { Total } \\
(\mathrm{N}=\mathbf{5 8 5})\end{array}$}} \\
\hline & \multicolumn{2}{|c|}{$\begin{array}{l}\text { Over weight } \\
(\mathrm{N}=441)\end{array}$} & \multicolumn{2}{|c|}{$\begin{array}{c}\text { Obese } \\
(\mathrm{N}=144)\end{array}$} & & \\
\hline & No. & $\%$ & No. & $\%$ & No. & $\%$ \\
\hline \multicolumn{7}{|l|}{ Using computer and internet at summer holiday } \\
\hline Daily & 413 & 70.6 & 134 & 23.0 & 547 & 93.5 \\
\hline 5 days/ week (week days) & 28 & 4.7 & 10 & 1.7 & 38 & 6.5 \\
\hline $\begin{array}{l}\text { Daily number of hours of using computer } \\
\text { and internet at summer holiday }\end{array}$ & $n=413$ & & $\mathrm{n}=134$ & & $(n=547)$ & \\
\hline$<4$ hours & 140 & 33.9 & 9 & 6.7 & 149 & 27.7 \\
\hline$\geq 4$ hours & 273 & 66.1 & 125 & 93.3 & 398 & 72.8 \\
\hline $\begin{array}{l}\text { Number of hours of using computer and } \\
\text { internet at summer holiday during } \\
\text { 5days/week }\end{array}$ & $\mathbf{n}=\mathbf{2 8}$ & & $\mathbf{n}=\mathbf{1 0}$ & & $(n=38)$ & \\
\hline$<4$ hours & 18 & 64.3 & 0 & 0 & 18 & 47.4 \\
\hline$\geq 4$ hours & 10 & 35.7 & 10 & 100 & 20 & 52.6 \\
\hline
\end{tabular}

Table (5): Distribution of overweight and obese students according to their watching TV and playing video games $($ No $=\mathbf{5 8 5})$.

\begin{tabular}{|c|c|c|c|c|c|c|}
\hline \multirow{3}{*}{ Items } & \multicolumn{4}{|c|}{ BMI } & \multirow{2}{*}{\multicolumn{2}{|c|}{$\begin{array}{c}\text { Total } \\
(\mathbf{n}=\mathbf{5 8 5})\end{array}$}} \\
\hline & \multicolumn{2}{|c|}{$\begin{array}{l}\text { Over weight } \\
(\mathrm{n}=441)\end{array}$} & \multicolumn{2}{|c|}{$\begin{array}{c}\text { Obese } \\
(n=144)\end{array}$} & & \\
\hline & No. & $\%$ & No. & $\%$ & No. & $\%$ \\
\hline \multicolumn{7}{|l|}{$\begin{array}{l}\text { Watching TV or playing video games on } \\
\text { school days }\end{array}$} \\
\hline No & \multirow{4}{*}{$\begin{array}{c}54 \\
223 \\
144 \\
20\end{array}$} & \multirow{4}{*}{$\begin{array}{c}9.2 \\
38.2 \\
24.6 \\
3.4 \\
\end{array}$} & \multirow{4}{*}{$\begin{array}{c}0 \\
130 \\
0 \\
14\end{array}$} & \multirow{4}{*}{$\begin{array}{c}0.0 \\
22.2 \\
0.0 \\
2.4 \\
\end{array}$} & 54 & 9.3 \\
\hline Daily & & & & & 353 & 60.3 \\
\hline Weekend(2days / week) & & & & & 144 & 24.6 \\
\hline 5 days/ week (week days) & & & & & 34 & 5.8 \\
\hline Daily & \multirow{3}{*}{$\begin{array}{c}\mathbf{n}=\mathbf{2 2 3} \\
117 \\
106\end{array}$} & \multirow{3}{*}{$\begin{array}{l}52.5 \\
47.5\end{array}$} & \multirow{3}{*}{$\begin{array}{c}\mathbf{n}=\mathbf{1 3 0} \\
5 \\
125\end{array}$} & \multirow[b]{3}{*}{$\begin{array}{c}3.8 \\
96.2\end{array}$} & $n=353$ & \\
\hline$<4$ hours & & & & & 122 & 34.6 \\
\hline$\geq 4$ hours & & & & & 231 & 65.4 \\
\hline Weekend(2days / week) & \multirow{3}{*}{$\begin{array}{c}\mathbf{n}=\mathbf{1 4 4} \\
0 \\
144\end{array}$} & \multirow{3}{*}{$\begin{array}{c}0.0 \\
100.0\end{array}$} & $\mathrm{n}=\mathbf{0}$ & \multirow{3}{*}{$\begin{array}{l}0.0 \\
0.0\end{array}$} & $\mathrm{n}=144$ & \\
\hline$<4$ hours & & & 0 & & 0 & 0.0 \\
\hline$\geq 4$ hours & & & 0 & & 144 & 100.0 \\
\hline 5 days/ week (week days) & \multirow{3}{*}{$\begin{array}{c}\mathbf{n}=\mathbf{2 0} \\
0 \\
0 \\
20\end{array}$} & \multirow{3}{*}{$\begin{array}{c}0.0 \\
100.0\end{array}$} & $\mathrm{n}=14$ & \multirow{3}{*}{$\begin{array}{c}0.0 \\
100.0\end{array}$} & $\mathrm{n}=34$ & \\
\hline$<4$ hours & & & 0 & & 0 & 00.0 \\
\hline$\geq 4$ hours & & & 14 & & 34 & 100.0 \\
\hline \multicolumn{7}{|l|}{$\begin{array}{l}\text { Watching TV or video games in summer } \\
\text { holidays }\end{array}$} \\
\hline Yes & \multirow{2}{*}{$\begin{array}{c}438 \\
3\end{array}$} & \multirow{2}{*}{$\begin{array}{c}74.9 \\
0.5\end{array}$} & \multirow{2}{*}{$\begin{array}{c}144 \\
0\end{array}$} & \multirow{2}{*}{$\begin{array}{c}24.6 \\
0.0\end{array}$} & 582 & 99.4 \\
\hline No & & & & & 3 & 0.6 \\
\hline $\begin{array}{l}\text { If you're watching TV or video games } \\
\text { on holidays summer }\end{array}$ & $n=438$ & & $n=144$ & & $\mathrm{n}=\mathbf{5 8 2}$ & \\
\hline$<4$ & 48 & 11.0 & 4 & 2.8 & 52 & 8.9 \\
\hline$\geq 4$ & 390 & 89.0 & 140 & 97.2 & 530 & 91.1 \\
\hline
\end{tabular}


Table (6): Distribution of overweight and obese students according to their practicing physical exercise $(\mathrm{No}=\mathbf{5 8 5})$.

\begin{tabular}{|c|c|c|c|c|c|c|}
\hline \multirow{3}{*}{ Items } & \multicolumn{4}{|c|}{ BMI } & \multirow{2}{*}{\multicolumn{2}{|c|}{$\begin{array}{c}\text { Total } \\
(\mathrm{N}=\mathbf{5 8 5})\end{array}$}} \\
\hline & \multicolumn{2}{|c|}{$\begin{array}{c}\text { Over weight } \\
(\mathrm{N}=441)\end{array}$} & \multicolumn{2}{|c|}{$\begin{array}{c}\text { Obese } \\
(N=144)\end{array}$} & & \\
\hline & No. & $\%$ & No. & $\%$ & No. & $\%$ \\
\hline \multicolumn{7}{|l|}{ Students' practicing physical exercise } \\
\hline Yes & 361 & 61.7 & 112 & 19.1 & 473 & 80.8 \\
\hline No & 80 & 13.7 & 32 & 5.5 & 112 & 19.2 \\
\hline $\begin{array}{l}\text { Mean hours of practicing } \\
\text { physical exercise per week }\end{array}$ & \multicolumn{2}{|c|}{$4.36 \pm 3.37$} & \multicolumn{2}{|c|}{$5.17 \pm 3.57$} & \multicolumn{2}{|c|}{$4.43 \pm 3.32$} \\
\hline Types of practiced physical exercise \# & \multicolumn{2}{|c|}{$(n=361)$} & \multicolumn{2}{|c|}{$(n=112)$} & \multicolumn{2}{|c|}{$(n=473)$} \\
\hline Running & 203 & 56.2 & 15 & 13.4 & 218 & 46.1 \\
\hline Swimming & 146 & 40.4 & 27 & 24.1 & 173 & 36.6 \\
\hline Basket ball & 177 & 49.0 & 12 & 10.7 & 189 & 39.9 \\
\hline Volley ball & 263 & 72.8 & 13 & 11.6 & 276 & 58.3 \\
\hline Foot ball & 165 & 45.7 & 50 & 44.6 & 215 & 45.5 \\
\hline Walking & 181 & 50.1 & 76 & 67.9 & 257 & 54.3 \\
\hline
\end{tabular}

\# Not mutually exclusive

Table (7): Distribution of overweight and obese students according to their eating habits. $(\mathrm{No}=\mathbf{5 8 5})$.

\begin{tabular}{|c|c|c|c|c|c|c|}
\hline \multirow{3}{*}{ Items } & \multicolumn{4}{|c|}{ BMI } & \multirow{2}{*}{\multicolumn{2}{|c|}{$\begin{array}{c}\text { Total } \\
(\mathbf{N}=585)\end{array}$}} \\
\hline & \multicolumn{2}{|c|}{$\begin{array}{c}\text { Over weight } \\
(\mathrm{N}=441)\end{array}$} & \multicolumn{2}{|c|}{$\begin{array}{c}\text { Obese } \\
(\mathrm{N}=144)\end{array}$} & & \\
\hline & No. & $\%$ & No. & $\%$ & No. & $\%$ \\
\hline \multicolumn{7}{|l|}{ Number of meals/day } \\
\hline Twice & \multirow{3}{*}{$\begin{array}{l}113 \\
227 \\
101\end{array}$} & \multirow{3}{*}{$\begin{array}{l}19.3 \\
38.8 \\
17.3 \\
\end{array}$} & \multirow{3}{*}{$\begin{array}{l}44 \\
52 \\
48\end{array}$} & \multirow{3}{*}{$\begin{array}{l}7.5 \\
8.9 \\
8.2\end{array}$} & 157 & 26.8 \\
\hline Three & & & & & 279 & 47.7 \\
\hline More than three & & & & & 149 & 25.5 \\
\hline \multicolumn{7}{|l|}{ Taking breakfast } \\
\hline Yes & \multirow{2}{*}{$\begin{array}{c}401 \\
40\end{array}$} & \multirow{2}{*}{$\begin{array}{c}68.5 \\
6.8 \\
\end{array}$} & \multirow{2}{*}{$\begin{array}{c}140 \\
4\end{array}$} & \multirow{2}{*}{$\begin{array}{c}24.0 \\
0.7\end{array}$} & 541 & 92.5 \\
\hline No & & & & & 44 & 7.5 \\
\hline \multicolumn{7}{|l|}{$\begin{array}{l}\text { Taking foods or beverages during } \\
\text { using computer or watching television }\end{array}$} \\
\hline Yes & \multirow{2}{*}{$\begin{array}{c}411 \\
30\end{array}$} & \multirow{2}{*}{$\begin{array}{c}70.3 \\
5.1\end{array}$} & \multirow{2}{*}{$\begin{array}{c}112 \\
32\end{array}$} & \multirow{2}{*}{$\begin{array}{c}19.1 \\
5.5\end{array}$} & 523 & 89.4 \\
\hline No & & & & & 62 & 10.6 \\
\hline If yes \# & $(n=411)$ & & $(n=112)$ & & $(n=523)$ & \\
\hline Fruit or vegetable & \multirow{5}{*}{$\begin{array}{c}114 \\
144 \\
206 \\
153 \\
89 \\
\end{array}$} & \multirow{5}{*}{$\begin{array}{l}27.7 \\
35.1 \\
50.1 \\
37.2 \\
21.7\end{array}$} & \multirow{5}{*}{$\begin{array}{l}30 \\
55 \\
75 \\
60 \\
40\end{array}$} & \multirow{5}{*}{$\begin{array}{l}26.8 \\
49.1 \\
67.0 \\
53.6 \\
35.7\end{array}$} & 144 & 27.5 \\
\hline Juices & & & & & 199 & 38.0 \\
\hline Entertainment & & & & & 281 & 53.7 \\
\hline Junk foods & & & & & 213 & 40.7 \\
\hline Tea,Coffee & & & & & 129 & 24.7 \\
\hline \multicolumn{7}{|l|}{$\begin{array}{l}\text { Eating the basic meals in front of the } \\
\text { TV or computer }\end{array}$} \\
\hline Yes & \multirow{2}{*}{$\begin{array}{l}261 \\
180\end{array}$} & \multirow{2}{*}{$\begin{array}{l}44.6 \\
30.8\end{array}$} & 107 & \multirow{2}{*}{$\begin{array}{c}18.3 \\
6.3\end{array}$} & 368 & 62.9 \\
\hline No & & & 37 & & 217 & 37.1 \\
\hline \multicolumn{7}{|l|}{ Number of eating junk food } \\
\hline Daily & \multirow{4}{*}{$\begin{array}{c}220 \\
97 \\
29 \\
95\end{array}$} & \multirow{4}{*}{$\begin{array}{c}37.6 \\
16.6 \\
5.0 \\
16.2\end{array}$} & 72 & \multirow{4}{*}{$\begin{array}{c}12.3 \\
4.3 \\
1.7 \\
6.3\end{array}$} & 292 & 49.9 \\
\hline Weekly & & & 25 & & 122 & 20.9 \\
\hline Monthly & & & 10 & & 39 & 6.6 \\
\hline Occasionally & & & 37 & & 132 & 22.6 \\
\hline
\end{tabular}

\# Not mutually exclusive 


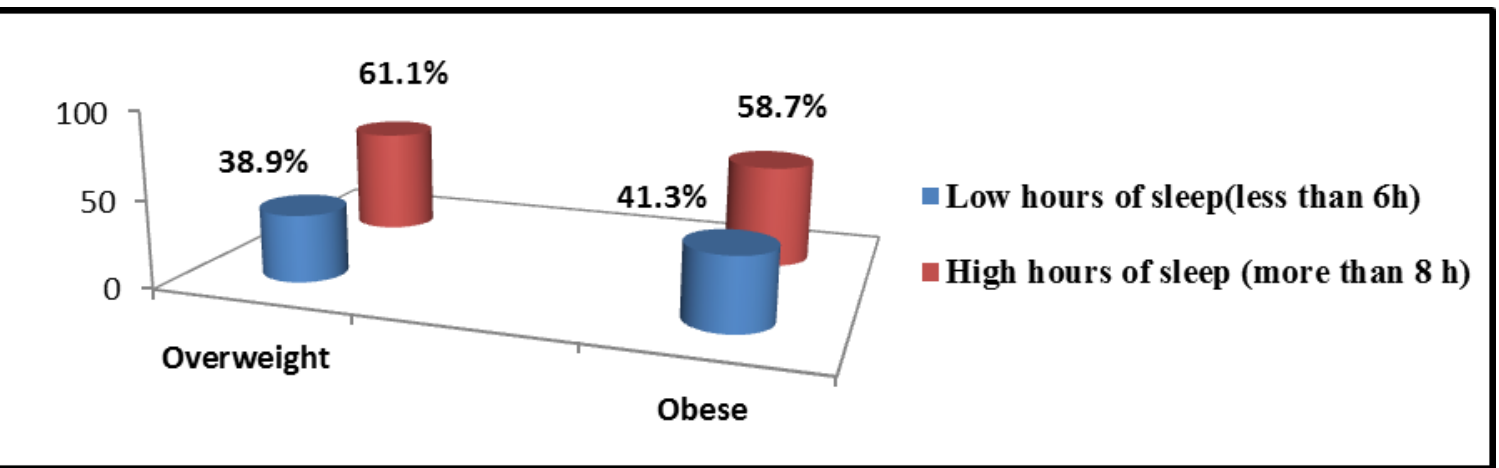

Figure (2): percentage distribution of sleeping hours / day among studied students. (No $=585)$.

Table (8): Distribution of overweight and obese students according to their health problems due to playing computer and watching TV. (No= 585).

\begin{tabular}{|l|c|c|c|c|c|c|}
\hline \multirow{2}{*}{ Items } & \multicolumn{4}{|c|}{ BMI } & \multicolumn{2}{c|}{$\begin{array}{c}\text { Total } \\
\text { (N=585) }\end{array}$} \\
\cline { 2 - 7 } & \multicolumn{2}{|c|}{$\begin{array}{c}\text { Over weight } \\
\text { (N=441) }\end{array}$} & \multicolumn{2}{c|}{$\begin{array}{c}\text { Obese } \\
\text { (N=144) }\end{array}$} & \multicolumn{2}{c|}{} \\
\cline { 2 - 7 } & No. & $\mathbf{\%}$ & No. & \% & No. & \% \\
\hline Health problems & & & & & & \\
\hline Yes & 244 & 41.7 & 140 & 23.9 & 384 & 65.6 \\
\hline No & 197 & 33.7 & 4 & 0.7 & 201 & 34.4 \\
\hline If yes\# & $\mathbf{n = 2 4 4}$ & & $\mathbf{n = 1 4 0}$ & & $\mathbf{n = 3 8 4}$ & \\
\hline Vision problems & 212 & 86.9 & 38 & 27 & 250 & 65.1 \\
\hline Headache & 187 & 76.6 & 30 & 21.4 & 217 & 56.5 \\
\hline Drowsy & 113 & 46.3 & 68 & 48.5 & 181 & 47.1 \\
\hline Hearing problems & 66 & 27.0 & 66 & 47.1 & 132 & 34.4 \\
\hline
\end{tabular}

\# Not mutually exclusive

Table (9): Stepwise Logistic Regression for predictable factors among studied students regarding overweight and obesity.

\begin{tabular}{|l|c|c|c|c|c|c|}
\hline \multicolumn{1}{|c|}{ Variables } & \multirow{2}{*}{ B } & \multirow{2}{*}{ S.E. } & \multirow{2}{*}{ Sig. } & \multirow{2}{*}{$\begin{array}{c}\text { Odds } \\
\text { Ratio }\end{array}$} & \multicolumn{2}{c|}{ 95\% C.I. for Odds Ratio } \\
\cline { 5 - 7 } \multicolumn{1}{|c|}{ Constant } & & & & Lower & Upper \\
\hline Mothers Education & -6.552 & .913 & .000 & .001 & & \\
\hline TV hours during school days & 2.092 & .465 & .000 & 8.103 & 3.258 & 20.156 \\
\hline Mother occupation & 3.592 & .516 & .000 & 36.322 & 13.209 & 99.880 \\
\hline Computer hours during summer & 1.585 & .348 & .000 & 4.878 & 2.468 & 9.640 \\
\hline
\end{tabular}

B: regression co-efficient

Table (1): Illustrates that the age of studied students ranged from 11 to 16 years old, with a mean age of $13.36 \pm 1.06$ years. Regarding students' academic year, approximately two thirds $(62.7 \%)$ of them were in grade one. While, minority $(16.6 \%)$ of the students were in grade 3 .

Table (2): Shows that slightly less than two thirds of students' fathers and mothers had university education (61.2\% and $61.3 \%$ respectively) and more than three quarters (79.2\%) of them had family income more than 1000 LE. It was observed that more than half $(57.3 \%)$ of mothers were housewives. Moreover, the mean crowding index of them was $1.63 \pm 0.85$ member/room.

Figure (1): Reveals that almost half (49\%) of the total studied students were overweight and $26 \%$ of them were normal weight compared to only $16 \%$ obese and only $9 \%$ were underweight. 
Table (3): Presents the distribution of overweight and obese students according to the presence of computer and internet at home. Among over weight and obese students, it was clear that, the majority $(98.5 \%)$ of the studied students had personal computers and nearly two thirds of them $(62.2 \%)$ had home internet. Of those students who had computer at home, nearly three quarters (73.9\%) of them were overweight and less than half $(42.9 \%)$ of those students who had home internet also were overweight. It was found that, students who had computer and internet at their home, they representing one quarter $(24.6 \%)$ and almost one fifth $(19.3 \%)$ respectively they were obese. In addition, $44.5 \%$ of those who didn't had computer at homes, went to net coffee (cyber) and relatives and neighbors to played computer.

Table (4): It was clear that ,approximately half (44.3\%) of all overweight/obese students and the majority $(93.3 \%)$ of them were using computer and internet daily in school days and summer holiday respectively. Also, it is observed that less than one third (29.9\%) of the students who using computer and internet daily in school days were overweight and more than one tenth (14.4\%) of them were obese. Moreover, about three quarters $(70.6 \%)$ of the students who were using computer and internet on daily basis in summer holidays were overweight and $23 \%$ of them were obese.

The same table reveals that, the majority $(86.5 \%)$ of all overweight and obese studied students and less than three quarters of them $(72.8 \%)$ were using computer and Internet 4 hours and more daily in school days and summer holidays respectively. Regarding to, students who using computer and Internet 4 hours and more daily in school days, the majority ( $86.9 \%$ \& $85.7 \%$ respectively) of them were overweight and obese. Furthermore, Of those students who using computer and Internet 4 hours and more daily in summer holiday, the majority $(93.3 \%)$ of them were obese and two thirds $(66.1 \%)$ of them were overweight.

Table (5): Illustrates that more than one third (38.2\%) of students who were watching TV or playing video games on daily basis at school days were overweight and nearly one quarter $(22.2 \%)$ of them were obese .Alternatively, about three quarters (74.9\%) of the students who were watching TV or playing video games on daily base in summer holidays were overweight and about one quarter $(24.6 \%)$ of them were obese.

Table (6): Shows that, the majority of overweight/obese students were playing physical exercises $(80.8 \%)$ and more than half $(54.3 \%)$ of them were walking. Unfortunately, from those who were playing physical exercises more than three fifths $(61.7 \%)$ of them were overweight and one fifth $(19.1 \%)$ were obese.

Table (7): Clarifies that, the majority $(89.4 \%)$ of these studied students taking foods or beverages during playing computer or watching television. In addition, from students had overweight and obese, less than two thirds (62.9\%) of them eating their basic meals in front of TV or computer. About less than half of them $(44.6 \%)$ were overweight and $18.3 \%$ were obese.

Figure (2): Reveals the sleeping hours' among overweight and obese students, it was clear that, approximately two thirds $(61.1 \%)$ of those students who were overweight and more than half $(58.7 \%)$ of those students who were obese had high sleeping hours.

Table (8): Illustrates health problems mentioned by the studied students associated with playing computers and watching T.V. wherever, less than two thirds $(65.6 \%)$ of all studied students who were overweight and obese, had health problems during playing computers and watching T.V. Of those who had health problems more than two fifths $(41.7 \%)$ of them were overweight and less than one quarter (23.9\%) were obese. Moreover highest percent (65.1\%) of students reported vision problems as a type of health problems associated with watching TV and using computer followed by headache $56.5 \%$ and drowsy $47.1 \%$.

Table (9): This table clarifies the outcomes of the stepwise logistic regression analysis for the conceivable risk factors of overweight/obesity. The analysis revealed that 4 variables were significant risk factors namely: mothers' education, TV watching hours during school days, mother occupation and computer using hours in summer. Lower education is a significant risk factor, wherever, the Odds Ratio $(\mathrm{OR})$ for this factor is 8.103 . This means that the risk of developing overweight and obese for the lower education group students is about 8 times that of the higher education group. Also, table reveals that, the risk for overweight and obesity among students watching TV for 4 hours or more during school days 
is more than 36 times in those watching TV $<4$ hours $(\mathrm{OR}=36.322)$.

The table also illustrates that overweight and obese is more prevalent for the non-working mothers and estimates that the involved risk is almost 5 times for their students $(\mathrm{OR}=4.878)$. Using computer hours during the summer shows a similar picture to watching TV hours during school days as seen from table (9) with about 17 times higher risk for the $\geq 4$ hours group $(\mathrm{OR}=16.608)$

\section{Discussion}

Adolescence follows childhood as the second most vulnerable stage in the life cycle. It is evident that, the majority of acquired harmful habits may affect adolescents' life time. Therefore, developing and maintaining healthy lifestyle behaviors is one of the factors that can help in extending life and enhancing health and vitality in later years (Lee et al., 2011).

Over weight /Obesity is one of the most serious public health challenges of the $21^{\text {st }}$ century. The prevalence has increased at an alarming rate (Ajie et al., 2014 \& Al-Hazzaa et al., 2012)

Although several genetic and lifestyle factors have been recognized to induce obesity, sedentary behaviours, including using of computer and watching $\mathrm{TV}$, have consider as potential risk factors for obesity since any child can be sedentary (Mosuwan et al ., 2014 \&Al-Ghamdi, 2013) Therefore, this study carried out to determine the association between using multimedia and overweight / obesity among the preparatory school students.

The World Health Organization maintains that the emerging trend of childhood obesity is rapidly affecting many middle and low income countries especially in urban settings. It is reported that of the forty-two million overweight children in the world, approximately thirty-five million reside in developing countries (WHO, 2011). The results of the existing study discovered that virtually half of the studied students were overweight. It may be attributed to increase of junk food intake related to response of food advertising (about half of students in current study eat junk food daily).This result is contradicted with study done in Qatar by Bener et al., (2012) and KSA by El Mouzan, (2010) who stated that less than one quarter of their studied sample were overweight. This discrepancy in results may be attributed to increase stander of living in theses Arab countries that allow population to follow healthy life style. Moreover the present study revealed that less than one fifth of students were obese. This is similar with study done in USA by Ogden, (2014) which stated that, $17 \%$ of youth in the USA are obese.

The use of multimedia (the Internet and Television) in many aspects of adolescence's life has become a widespread global phenomenon, just about $90 \%$ of adolescents practice the Internet and communications gears (Ajie et al., 2014 \& Straker et al., 2009). It was noticed from the current study that, almost all of the studied students had personal computers and nearly two thirds of them had home internet. It may be attributed to many reasons as, in Egypt, most computer devices sold by instalment, school activities done on computers in most private and governmental schools and increase types of software and games to attract them in using internet. In addition, it considered a cheap recreational method for many children and adolescents. The finding of the present study is in agreement with study done by File, (2013), \& Madden et al .,(2013) who stated that the majority of teens had a personal computer. On the contrary to study done in Ghana by Quarshie, (2012) which revealed that $32 \%$ and $56.7 \%$ of the school children do not have entree to the computer and internet at home respectively. This finding could be interpreted in the light of the fact that policy makers in education in Ghana have hailed the introduction of Information and Communication Technology (ICT) in Ghanaian schools as amazing step that will donate to knowledge manufacture, communication and information allocation among students in the school system (The Ghana Information Communication (ICT) in education, 2006)

The American Academy of Pediatrics (AAP) stated that, internet use guidelines for adolescents to overcome weight gain, recommend two hours only per day for computer and internet habit (Moreno et al., 2013). The present study revealed that, the highest percent of students who using computer and internet 4 hours and more either in school days and summer holidays were overweight and obese students. It may be attributed to that extended unwarranted Internet use and TV viewing receipts away appreciated time from certain compulsory activities that children should engage in. Moreover, extensive exposure to computer and TV may increase consumption of food.

Likewise, the current study revealed that students spent their time on computer during summer for 4 hours and more had 16.6 times risk for overweight and obesity than those with $<4$ hours. It is evident that prolonged excessive playing computers and watching TV takes away valuable time from certain necessary activities that adolescents should engage in. These results supported with many studies done in Portugal by Mota et al., (2006) which stated that, adolescents who used computers more than 4 hours per day were likely (odds ratio: 5.79 ; $\mathrm{p}<.003$ ) to be overweight or obese and also study done in UAE by Musaiger et al., (2003) who found that watching TV 
for $>4$ hours per day increased the relative risk of obesity by 1.3 among students in the UAE. Moreover, in a Scandinavian study, Andersen et al., (2005) reported that the odds ratio of becoming obese for school children watching TV/computer screens for a period of $>4$ hours per day was 2.5 times higher relative to those watching for $<1$ hour per day. These results are contrary to study done in USA by Moreno et al., (2013) which illustrated negative association between Internet use among adolescent and number of hours /day they spent on computers.

It is universally accepted that media-based sedentary behaviors, such as TV viewing and video game playing, contest for time that would otherwise be spent in physical activity, which might bring about obesity, for instance, TV viewing has been acknowledged as an incentive for unwarranted eating and deskbound behavior, and the prevalence of obesity is lowest among children watching 1 or fewer hours of television per day and highest among those watching 4 or more hours of television per day

(Tremblay al., 2006) The present study showed that, nearly two thirds of the studied students watching television or playing video games daily during school days. In addition, almost all students watching T.V and playing video games during summer holidays. These results, similar to study done in USA by Vandewater et al., (2004), Thailand by Mo-suwan et al., (2014), and KSA by Al-Ghamdi,(2013) who confirmed that ,watching television during adolescents is associated with an increased BMI. On the other hand, McMurray et al., (2000) originate that there was no connotation between television or video game use and BMI in a sample of 2389 adolescents. Odds ratio of flattering overweighed and obese for students using Television watching hours at school days was 36 times higher risk for 4 hours and more relative to $<4$ hours. A study from Taiwan, done by Johnson et al., (2004), reports similar results. Moreover, study done in Egypt by Bassiouny \& Madian, (2013) reported that nearly two thirds of adolescence spend their leisure time in watch TV.

It has been challenging to validate persuasively that physical activity plays a momentous role in the progress of excess body fat during childhood. From the energy balance point of view, an inverse relationship between activity levels and obesity in children should be expected (Straker et al., 2009 \& Carter, 2006). Whereas, some studies support the traditional view that physical activity is inversely associated with obesity. Dionne et al., (2000) another abortive to display evidence of such a connection Kelley et al., (2015). Nonetheless, the present study showed that more than three quarters of all studied students were playing sports and more than half of them walking on daily basis. Unfortunately, from those who were playing sports and walking almost half of them were overweight. This may be attributed clearly that, the hours spent watching TV are sedentary hours that are not spent being active. These results in accordance with study done in Portuguese adolescent, who did not show differences in reported levels of physical activity according to the BMI groups (Mota et al., 2006)

In divergence with these results, international studies done by, Graves et al., (2007), Maddison et al., (2007), Straker et al., (2007) \&Foster et al., (2006) created a strong association between level of physical activities and weight gain. It may be attributed to culture different in which students in developed countries affianced to regular protective exercise training in order to overcome weight gain.

Wiecha et al., (2006), conveyed that each hour increase in TV viewing was concomitant with supplementary $167 \mathrm{kcal} /$ day and with increases in consumption of foods publicized on TV. Moreover, Barr et al., (2009) \& Utter et al., (2006) and recounted that the longer the exposure to $\mathrm{TV}$, the more commercials viewed, which clues to increased consumption of foods high in sugar, fat and salt. The present study bared that, less than half of those students who eating their basic meals in front of TV or computer were overweight and more than one fifth of them were obese. This is in harmony with Smetanina et al., (2015) which highlights the fact that TV watching, especially during the meals, and the presence of a TV in the child's bedroom, has been reported to be associated with overweight and obesity.

The current study revealed that, the slightly less than two thirds of all studied students suffered from health problems during using computer and watching TV. Of those who had health problems, majority of students who had vision problems were overweight and slightly more than one quarter of them were obese. This result congruent with study done by American Optometric Association at 2011 on school age students to document the relationship between low vision and time spent on TV/computer screens by children. (American Optometric Association, 2011) All of the major predictors of overweight and obesity found in this study as mothers' education, TV hours during school days, mother work and computer hours in summer were similar to those found in other international studies (Moreno et al., 2013). Nevertheless, theses predictors not accordance on study done in Qatar by Bener et al., (2012) in which, predictors included less physical activity, less sleeping hours, family income, frequency of watching TV/Computer/Video Game, age and number of bedrooms. 
On the other hand, Study done in India by Anuradha, (2015) stated that, adolescents belonging to educated mother show higher rates of overweight and obesity. In accordance with these results, the current study stated that, the risk of developing overweight and obese for the lower education group students is about 8 times that of the higher education group adolescents with lower educated mother had 8 times to get overweight and obesity than adolescents with educated mother. This is chiefly attributed to other issues like deficiency of awareness, peculiar dietary outlines with more energy dense foods.

Researches done, in North America and Europe. Hong, (2011) \& Ziol-Guest, (2013) showed that longer working hours of mothers increased BMI of their children. These results are contrary with the current study which indicated that adolescents with non-working mother had 4.8 times to get overweight and obesity than adolescents with working mother. It may be attributed to difference in nature of food and methods of cooking in addition to non-working mother had time and chance to serving many types of foods than employed mothers.

Adolescent's necessity to be treated as a separate sector of our population and the families of them can demonstrate to be a great basis to help their adolescents to animate a health life. The study has endeavored to highlight several areas of apprehension with respect to adolescent lifestyle. Moreover, increase their awareness regarding risk behaviors in order to maintain their wellbeing and promote happy life (Saffari \& Amini 2013).

In study done among Indian school children by Kameswararao \& Bachu's (2009), they found that by increasing physical activity and decreasing time spent watching TV, sedentary lifestyle and the consumption of high sugar, carbohydrate and fatty foods, could be reduced significantly to help in overcome weight advance among adolescent.

As adolescents devote increasing amounts of time in front of computer monitors (in accumulation to the time spent in front of a television screen), they are probable to increase their risk of weight gain. (Blass, 2006) In the present study, this was inveterate, especially with regard to the time spent over the weekend. Consequently, the American Academy of Pediatrics recommends parents to limit the time their children spend with the media and to highlight alternative activities, such as sports events, physical conditioning, and imaginative play (American Academy of Pediatrics.2011)

\section{Conclusion \& Recommendations}

The present study reveals that, there was an association between using multimedia and overweight / obesity among the preparatory school students. Moreover, mother education and occupation, TV watching hours in school days and computer using in summer holidays signifies imperative risk aspects for overweight / obesity in adolescence. .

So, based on the results of the present study, it is recommended that, 1) School-based intervention programs should be implemented to increase children's awareness regarding negative effects of long exposure to watching $\mathrm{TV}$ and playing computers. 2) Health education programs also should directed to parents in order to play a more positive role in reducing the time their children spend watching TV and encourage them to engage in physical activities.

Acknowledgment

Our deep gratitude goes to our study subjects who volunteered and give us all the relevant information. Last but not least, we would like to thank the staff of school administrators for their cooperation and help during data collection

\section{References}

1. Ajie, W., \& Chapman Novakofski, A., (2014): Impact of computer-mediated, obesity-related nutrition education interventions for adolescents: a systematic review, Journal of Adolescent Health, Vol. (54), Pp .631-45.

2. Al-Ghamdi, S., (2013): The association between watching television and obesity in children of school-age in Saudi Arabia, Journal of Family and Community medicine,Vol.(20),No. (2).Pp. 83-9.

3. Al-Hazzaa, H., Abahussain, N., Al-Sobayel, H., Qahwaji, D., \& Musaiger, A., (2012): Lifestyle factors associated with overweight and obesity among Saudi adolescents, BMC public health, Vol.(12),Pp.1-12.

4. American Academy of Paediatrics (2011): Committee on Public Education. Children, adolescents, and television. Paediatrics,; 107:423-6.

5. American Optometric association. Impact of computer use on children's vision .2011. Available from:

6. www.aoa.org/x5379.xml.

7. Andersen, L., Lillegaard, I., Overby, N., Lytle, L., Klepp, K., Johansson, L., (2005): Overweight and obesity among Norwegian

8. School children: changes from 1993 to (2000): Scandinavian Journal of Public Health Vol. (33), P.p.99-106.

9. Anuradha, R., Sathyavathi, R., Muneeswara, R., Hemalatha, G., \& Geetha, S., Reddy K ., (2015): Effect of social and environmental 
determinants on overweight and obesity prevalence among adolescent school children, Indian J Endocrinal Metab, Vol.(19),No.(2).Pp. 283-7.

10. Barr-Anderson, D., Larson, N., Nelson, M., Neumark-Sztainer, D., \& Story, M., (2009): Does TV viewing predict dietary intake five years later in school students and young adults? , Int J Behav Nutr Phys Act,Vol.( 30),Pp. 6-7.

11. Barton, M., (2010): Screening for obesity in children and adolescents: US preventive services task force recommendation statement, Pediatrics, Vol. (125), No. (2).Pp. 361-7.

12. Bassiouny R., \& Madian A., (2013): Healthy life style in adolescence - Issue and challenge for the Egyptian health sector. Fourth international nursing conference, Jordanian nursing council .Amman - Jordan.

13. Bener, A., Al-Mahdi, H ., Al-Nufal, M., Ali, A., Vachhani, P., \&Tewfik, I., (2012): Association between childhood computer use and risk of obesity and low vision, PHF, Vol.(1),No.(3).Pp. 66-72.

14. Bener, A., Al-Mahdi, H., Vachhani, P., AlNufal, M., \& Ali, A.,(2010): Do excessive internet use, television viewing and poor lifestyle habits affect low vision in school children?, Journal of Child Health Care, Vol.(14),No.(4).Pp. 375-85.

15. Blackwell, L., (2015): Influence on children media - history of media for children, general considerations, studies of media influence, domains of influence, Recommendations. Available at: http://education.stateuniversity.com/pages/2212/ Media-Influence-onhildren.html\#ixzz3SU6CERS7

16. Blass, E., Anderson, D., Kirkorian, H., Pempek, T., Price, I., \& Koleini M., (2006): On the road to obesity: Television viewing increases intake of high- density foods, Physical Behav,Vol.( 88),Pp.597-604.

17. Carter, O., (2006): The weighty issue of Australian TV food ads and childhood obesity, Health Promot J Austr, Vol.(17),No.(1).Pp. 511.

18. Centres for disease control \& prevention. Division of nutrition, physical activities and obesity .2015. Available at: www.cdc.gov/healthy.

19. Dionne, I., Alméras, C., \& Bouchard, A.,(2000): The association between vigorous physical activities and fat deposition in male adolescents, Med. Sci. Sports Exerc, Vol.(32),No.(2).Pp.392- 4.
20. El Mouzan, M., Foster, P., Al Herbish, A., Al Salloum, A., Al Omer, A., Qurachi, M., \& Kecojevic, T., (2010): Prevalence of overweight and obesity in Saudi children and adolescents, Ann Saudi Med, Vol.(30),No.(3).Pp. 203-8.

21. File, T., (2013): Computer and internet use in the United States, Current Population Survey Reports, Available at: https://www.census.gov/prod/2013pubs/p20569.pdf

22. Foster, K., Aaras, A., \&Lie, I., (2006): Work with visual display units: long-term health effects of high and downward line-of-sight in ordinary office environments, International Journal of Industrial Ergonomics, Vol. (36),Pp. 331-43.

23. Graves, L., (2007): Comparison of energy expenditure in adolescents when playing new generation and sedentary computer games: cross sectional study, British Medical Journal, Vol. (335), Pp. 1282-4.

24. Hong, J., (2011): Decomposition of changes in labor force participation of married women: 1998-2007, Women's Stud, Vol. (80), Pp.14380.

25. Johnson, G., Cohen, P., Kasen, S., First B., \& Brook S., (2004): Association between television viewing and sleep problems during adolescence and early adulthood, Arch Pediatr Adolesc, Vol. (2),Pp. 597-8.

26. Kameswararao, A., \& Bachu, A., (2009): Survey of childhood diabetes and impact of school level educational interventions in rural schools in Karimnagar district (India), Int $\mathbf{J}$ Diabetes Dev Ctries,Vol.( 29),No.(2).Pp. 69-73.

27. Kelley, G., Kelley,K.,\&Pate, R., (2015): Exercise and BMI in Overweight and Obese Children and Adolescents: A Systematic Review and Trial Sequential Meta-Analysis, BioMed Research International,Vol.(2015).Pp.7.

28. Lee, R., \& Loke, A., (2011): Lifestyle behaviors and psychosocial well-being of Chinese adolescents in Hong Kong and Guangzhou, China: a cross-sectional comparative survey, J Clin Nurs,Vol.(20) .Pp.2733-43.

29. Madden, M., Lenhart, A., (2013): Duggan M, Cortesi S, Gasser U. Teens and Technology. Available at: http://www.pewinternet.org/Reports/2013/Te ens-and-Tech.aspx

30. Maddison, R., (2007): Energy expended playing video console games: an opportunity to 
increase children's physical activity?, Paediatric Exercise Science ,Vol. (19).Pp. 1-24.

31. Manyanga, T., El-Sayed, H., Doku, D., \& Ranall, J., (2014): The prevalence of underweight, overweight, obesity and associated risk factors among school-going adolescents in seven African countries, BMC public health,Vol.(14),Pp.887.

32. McMurray, R., Harrell, J., Deng, S., Bradley, C., Cox, M., \& Bangdiwala, S., (2000): The influence of physical activity, socioeconomic status, and ethnicity on the weight status of adolescents, Obesity Research, Vol. (8) .Pp.130-1.

33. Mo-suwan, L., Nontarak, J., Aekplakorn, W., \& Satheannoppakao, W., (2014): Computer game use and television viewing increased risk for overweight among low activity girls: fourth Thai. Health examination survey 2008-2009, International Journal of Pediatrics, Vol. (3) .Pp.1-7.

34. Moreno, M., Jelenchick, L., Koff, R., Eickhoff, J., \& Davis, A.,(2013): Associations between internet use and fitness among college students: an experience sampling approach, Journal of Interaction Science, Vol. (1),No.(4).Pp. 2-8.

35. Mota ,J.,\& Ribeiro, j.,(2006): Obesity, physical activity, computer use, and TV viewing in Portuguese adolescents, Pediatric Exercise Science, Vol.( 17),Pp.113-21.

36. Musaiger, A., Lloyd, O., Bener, A., \& AlNeyadi, S., (2003): Lifestyle factors associated with obesity among male university students in the UAE, Nutrition \& Food Science, Vol. (33),Pp. 145-7.

37. Ogden, C., Carroll, M., Kit, B., \& Flegal, K.,(2014):Prevalence of childhood and adult obesity in the United States, 2011-2012,JAMA, Vol.( 26),No. (8).Pp.806-14.

38. Quarshie, H., (2012): The impact of computer technology on the development of children in Ghana, Journal of Emerging Trends in Computing and Information Sciences, Vol.(3),No.(5) .Pp.717-22.

39. Rosen, L., Lim, J., Felt, L., Carrier, A., Cheever, M., \& Rokkum, L., (2014): Media and technology use predicts ill-being among children, preteens and teenagers independent of the negative health impacts of exercise and eating habits, Computers in Human Behavior, Vol.( 35).Pp. 364-75.

40. Saffari, M.,\& Amini, N., (2013): Educational intervention on health related lifestyle changes among Iranian adolescents, Iranian $\mathrm{J}$ Pub Health, Vol.( 42 ),No.(2).Pp.172-81.
41. Singh, K., Siahpush, M., \& Kogan, M., (2010): Neighborhood socioeconomic conditions, built environments, and childhood obesity, Gopal. Health affairs, Vol. (29), No. (3).Pp. 503-12.

42. Smetanina, N., Albaviciute, E., Babinska V., Karinauskiene, L., Albertsson-Wikland K., Petrauskiene, A., \& Verkauskiene, R., (2015): Prevalence of overweight/obesity in relation to dietary habits and lifestyle among 7-17 years old children and adolescents in Lithuania, BMC Public Health, Vol.(15),Pp.1001-12.

43. State information services: (2014): gateway to Egypt; central agency of public mobilization and statistics of El-Beheira governorate, books of census and statistics.. Retrieved at www.sis.gov.eg.

44. Straker, L., \& Abbott, R., (2007): Effect of screen based media on energy expenditure and heart rate in 9- to 12- year old children, Pediatric Exercise Science, Vol. (19),Pp. 45971.

45. Straker, L., Pollock, C., \&Maslen, B., (2009): Principles for the wise use of computers by children. Ergonomics, Vol. (52), No. (11).Pp. $1386-401$.

46. Talat, M.,\&EIShahat, E., (2016): Prevalence of overweight and obesity among preparatory school adolescents in Urban Sharkia Governorate, Egypt, Vol.(64),No.(1).Pp.

47. Tewfik, I., (2011): Obesity and low vision as a result of excessive internet use and television viewing, International Journal of Food Sciences and Nutrition, Vol. (62), No. (1).Pp. 60-2.

48. The Ghana Information (2006): Communication (ICT) in education policy

49. The Royal Australasian College (2004): of Physicians: Paediatrics \& Child Health Division. Paediatric Policy: Children and the media: Advocating for the future.. Available at: https://www.racp.edu.au/docs/defaultsource/advocacy-library/children-and-themedia-advocating-for-the-future.pdf.

50. Tremblay, S., \& J., Willms. (2006): Is the Canadian childhood obesity epidemic related to physical inactivity?, Int. J. Obes, Vol.( 21),Pp. $1100-5$.

51. United Nations Children Fund (2015): (UNICEF). Children in Egypt: Statistical Design: youth and adolescence . Available at: www.unicef .org /Egypt.

52. Utter, J., Scragg, R ., \& Schaaf, D., (2006): Associations between TV viewing and consumption of commonly advertised foods among New Zealand children and young 
adolescents, Public Health Nutr, Vol.(9),No.(5).Pp. 606-12.

53. Vandewater, E., Shim, M., \&Caplovitz, A., (2004): Linking obesity and activity level with children's television and video game use ,Journal of Adolescence ,Vol.( 27),Pp .71-85.

54. Wiecha, J., Peterson, K., Ludwig, D., Kim, J., Sobol, A., \&Gortmaker, S., (2006): When children eat what they watch: Impact of TV viewing on dietary intake in youth, Arch Pediatr Adolesc Med, Vol.( 160),No.(4).Pp. 436 - 42.

55. Wieting, M., (2008): Cause and effect in childhood obesity: solutions for a national epidemic, J Am Osteopath Assoc, Vol. (108),Pp.545-52.

56. World Health Organization .10 Facts on adolescent health. 2009. Available at http // www.who.int/adolescent health.

57. World Health Organization, (2011): Childhood overweight and obesity.. Available at http://www.who.int/dietphysicalactivity/childho od/en

58. Ziol-Guest, K., Dunifon, R., \& Kalil, A., (2013): Parental employment and children's body weight: mothers, others, and mechanisms, Soc Sci Med,Pp. 95:52-9. 